\title{
CME/Antworten: Traumatisierte Geflüchtete in der Hausarztpraxis - ein Kurzleitfaden
}

\author{
CME/Answers: Traumatized Refugees in Family Practice - \\ A Quick Reference Guide
}

Matthis Schick

Ambulatorium für Folter- und Kriegsopfer, Klinik für Konsiliarpsychiatrie und Psychosomatik, Universitätsspital Zürich, Zürich

Zusammenfassung: Traumatische Erfahrungen, Flucht und das Leben im Exil stellen per se keine Krankheit dar, gehen jedoch mit einem erhöhten psychischen Erkrankungsrisiko einher. Die Hausarztpraxis bleibt angesichts des Mangels an spezialisierten Therapieplätzen oft die einzige Behandlungsstelle für traumatisierte Geflüchtete. Besonders herausfordernd ist die Kombination von komplexen Beschwerdebildern, mehrdimensionalen psychosozialen Belastungssituationen und erschwerten Behandlungsbedingungen, welche im dichten Praxisalltag betriebswirtschaftlichen und organisatorischen Grenzen gegenüberstehen. Im Artikel werden Empfehlungen für den Umgang mit dieser heterogenen Patientengruppe diskutiert.

Schlüsselwörter: Trauma, Geflüchtete, Exil, Therapie

\begin{abstract}
Traumatic experiences, flight and life in exile do not constitute an illness per se, but are associated with an increased risk of mental illness. In view of the lack of specialized treatment units, the general practitioner's office often remains the only place of treatment for traumatized refugees. Particularly challenging is the combination of complex complaints, multidimensional psychosocial stress factors and difficult treatment conditions which face managerial and organizational limitations in the dense daily practice routine. In this article, recommendations for working with this heterogeneous group of patients are discussed.
\end{abstract}

Keywords: Trauma, refugees, exile, therapy

Frage 1: Welche Aussagen zur psychischen Gesundheit von Geflüchteten treffen zu? (Mehrfachauswahl)

a) Geflüchtete und MigrantInnen ohne Fluchthintergrund haben ein vergleichbares psychisches Erkrankungsrisiko.

b) Die psychische Gesundheit von Geflüchteten hängt mit vielen Faktoren zusammen, u.a. mit früheren traumatischen Erfahrungen, aber auch mit post-migratorischen Belastungsfaktoren.

c) Psychische Erkrankungen können die Integrationsfähigkeit von Geflüchteten stark beeinträchtigen.

d) Unbehandelte psychische Erkrankungen weisen ein hohes Chronifizierungsrisiko auf, frühe Diagnosestellung und Behandlung sind daher wichtig.

e) In Ermangelung ausreichender spezialisierter Behandlungsplätze kommt den Hausärztinnen und Hausärzten eine hohe Bedeutung in der Versorgung dieser Patientengruppe zu.

Antworten b), c) und e) sind richtig.
Ad b): Multidimensionale, bio-psycho-soziale Belastungssituationen finden sich bei Geflüchteten sehr häufig als Mitursache psychischer Erkrankungen.

Ad c): Korrekt: Psychische Erkrankungen gehen oft mit kognitiven, interpersonellen und anderen Beeinträchtigungen einher, die die Integration stark erschweren können. Ad e): Korrekt: Allerdings gibt es dafür auch andere Gründe, z.B. das psychiatrische Stigma. Dennoch sollte der Einbezug von Psychiatrie und spezialisierten Zentren nicht unversucht bleiben.

Ad a): Das psychische Erkrankungsrisiko von Geflüchteten ist gegenüber Migrant_innen ohne Fluchthintergrund erhöht.

Ad d): Dies ist nicht nur bei Geflüchteten so, dort aber schwieriger anzugehen.

Frage 2: Welche der folgenden Aussagen zur Verständigung ist falsch? (Mehrfachauswahl)

a) Der Einbezug von Laiendolmetschenden führt im Vergleich zu qualifizierten Dolmetschenden häufiger zu medizinisch relevanten Missverständnissen. 
b) Sprachliche Verständigungsschwierigkeiten entbinden nicht von der ärztlichen Aufklärungs- und Informationspflicht.

c) Der Einbezug von qualifizierten Dolmetschenden an sich garantiert noch keine ausreichende inhaltliche und/oder transkulturelle Verständigung.

d) Wenn die Patientin / der Patient eine Vertrauensperson zum Dolmetschen mitbringt, kann man davon ausgehen, dass man diese Person ohne Bedenken fürs Dolmetschen einsetzen kann.

e) Qualifizierte Dolmetschende können wertvolle kulturelle Kontextinformationen beisteuern, welche die medizinische Beurteilung erleichtern.

\section{Antworten a), b), c) und e) sind richtig.}

Ad a): Laiendolmetscher machen häufiger Übersetzungsfehler, Fehlinterpretationen und Auslassungen, und auch die PatientInnen bringen sensible Informationen weniger zur Sprache, was Fehlbeurteilungen nach sich ziehen kann. Ad b): Der Gesetzestext sieht keine Ausnahme für Fremdsprachige vor.

Ad c): Auch bei qualifizierten Dolmetschenden kann es wenn auch seltener - zu Missverständnissen kommen, was in der Behandlung stets mitbedacht und ggf. überprüft werden sollte.

Ad e): Dolmetschende können in den Vor- und Nachgesprächen die kulturelle Binnenperspektive einbringen. Die Interpretationshoheit sollte jedoch bei den Behandelnden bleiben.

Ad e): Sogenannte «Vertrauenspersonen» werden oft nicht aufgrund ihrer Vertrauenswürdigkeit rekrutiert, sondern ihrer Verfügbarkeit und Sprachkenntnisse wegen.

Frage 3: Welche der folgenden Aussagen zu traumatischen Erfahrungen sind richtig? (Mehrfachauswahl)

a) Traumatische Erfahrungen führen nicht zwangsläufig zu psychischer Erkrankung.

b) Psychische Traumatisierungen äussern sich primär in Form einer posttraumatischen Belastungsstörung.

c) Ob eine psychische Traumafolgestörung einer spezifischen Behandlung zugeführt werden soll, hängt vom Leidensdruck, dem Beeinträchtigungsgrad und der Bereitschaft zu einer Behandlung ab.

d) Traumatische Erfahrungen und daraus resultierende psychische Beschwerden werden meist nicht spontan vorgebracht.

e) Aus einem abgewiesenen Asylgesuch kann nicht auf das Fehlen von traumatischen Erfahrungen oder psychischen Traumafolgestörungen geschlossen werden.

\section{Antworten a), c), d) und e) sind richtig.}

Ad a): Das psychische Erkrankungsrisiko steigt mit Anzahl, Dauer und interpersonellem Charakter der traumatischen Erfahrungen.

Ad c): Trauma-spezifische Therapieverfahren können sehr hilfreich sein, erfordern aber Auseinandersetzungsbereitschaft und gewisse Belastungsreserven. Druck führt zu erhöhten Abbruchraten.
Ad d): Sowohl interpersonelle Traumata als auch psychische Beschwerden werden oft erst nach längerem Vertrauensaufbau eröffnet.

Ad e): Traumatische Erfahrungen sind kein juristisches Kriterium hinsichtlich Erfüllung der Flüchtlingseigenschaft. Ein prophylaktisch geflohener Journalist kann ohne jegliche Traumatisierung als Flüchtling anerkannt werden, während ein schwer traumatisierter Asylsuchender aus Syrien u.U. einen Negativentscheid bekommt.

Ad b): Die posttraumatische Belastungsstörung ist zwar eine häufige, jedoch bei weitem nicht die einzige mögliche Traumafolgestörung. Neben anderen kommen auch Depression, Angst-, Zwangs-, somatoforme Störungen oder Substanzabusus sowie deren Kombinationen vor.

\section{Frage 4: Welche Aussagen zu Behandlungsbarrieren} treffen zu? (Mehrfachauswahl)

a) Das hohe Stigma psychischer Erkrankungen kann die Offenlegung psychischer Symptome erschweren oder verhindern.

b) Gewisse traumatische und/oder das Ehrgefühl verletzende Erfahrungen können bei Bekanntwerden massive soziale Sanktionen, im Extremfall bis hin zum Ehrenmord, nach sich ziehen.

c) Transkulturell divergierende Vorstellungen und Erwartungen hinsichtlich Symptompräsentation, Erkrankung und Behandlung können zu Missverständnissen, Fehlbeurteilungen und in der Folge zu Malcompliance führen.

d) Mangelnde Health Literacy kann sich u.a. in fehlender Geltendmachung von Beschwerden, fehlender Inanspruchnahme von Behandlung und ungünstigem Gesundheitsverhalten manifestieren.

e) Der chancengleiche Zugang zu Gesundheitsdienstleistungen ist in der Schweiz für alle Krankenversicherten gewährleistet.

Antworten a), b), c) und d) sind richtig.

$\mathrm{Ad}$ a): nicht nur bei Geflüchteten.

Ad b): Die Kosten einer Offenlegung können deren möglichen Gewinn bei weitem übersteigen. Bisweilen werden solche Aspekte sogar im Asylverfahren verschwiegen.

Ad c): Die Perspektivenumkehr - wir als Patient_innen bei einer/einem traditionellen Heiler_in - macht das Dilemma unmittelbar nachvollziehbar.

Ad d): Wer z.B. nie von einer posttraumatischen Belastungsstörung gehört hat, kann eine solche auch nicht geltend machen.

Ad e): Der chancengleiche Zugang zu Gesundheitsdienstleistungen ist lediglich theoretisch gegeben, wird bei Geflüchteten praktisch aber oft durch Behandlungsbarrieren wie z.B. Verständigungsschwierigkeiten verhindert.

Frage 5: Welche Aussage zu zwischenmenschlichen Traumatisierungen wie Folter oder anderen Menschenrechtsverletzungen ist falsch? (Mehrfachauswahl) a) Zwischenmenschliche Traumatisierungen können sich nicht nur auf Körper und Psyche, sondern auch auf 
Wertesystem, Selbst- und Weltbild, Vertrauens- und Beziehungsfähigkeit auswirken.

b) Je früher traumatische Erfahrungen biografisch einsetzen und je länger sie anhalten, desto mehr wird die gesamte Persönlichkeitsentwicklung mitbetroffen.

c) Menschenrechtsverletzungen gehen oft mit einer hohen Sensibilität gegenüber realer oder vermeintlicher Ungerechtigkeit oder Ungleichbehandlung einher, was in der Zusammenarbeit berücksichtigt werden sollte.

d) Folter ist so brachial, dass sie sich auch körperlich nachweisen lässt.

e) Ausreichend Zeit für den Vertrauensaufbau ist ein Schlüsselelement der Behandlung.

\section{Antworten a), b), c) und e) sind richtig.}

Ad a): Gerade die zwischenmenschlichen Beeinträchtigungen können sich gravierend auf (soziale) Funktionsfähigkeit, Partizipation und Lebenszufriedenheit auswirken. Ad b): Nicht nur bei Geflüchteten.
Ad c): Die Erfahrung prägt die Wahrnehmung, schon eine neutrale Haltung kann als distanziert oder feindselig wahrgenommen werden. Ein besonderes Augenmerk auf Freundlichkeit und Respekt unterstützt v.a. in der Anfangsphase den Vertrauensaufbau.

Ad e): Vertrauen lässt sich nicht einfordern oder erzwingen, sondern entwickelt sich günstigenfalls aus einem Prozess.

Ad d): Oft zielt Folter darauf ab, keine nachhaltig sichtbaren Spuren zu hinterlassen. Die psychologischen Auswirkungen sind deswegen nicht geringer.

\section{PD Dr. med. Matthis Schick}

Leitender Arzt

Universitätsspital Zürich, Klinik für Konsiliarpsychiatrie und Psychosomatik

Culmannstrasse 8

8091 Zürich

matthis.schick@usz.ch,www.psychiatrie.usz.ch 